\title{
GENETICS
}

\section{The crystal ball clears for breast cancer therapy?}

G iven the range of treatment choices and the difference in response to treatment, the search for a tool to predict response when patients are newly diagnosed with breast cancer has been intense. If a way to 'see into the future' could be found, it would remove a lot of the uncertainty in therapy choice.

For patients with HER2-positive cancer the obvious first treatment choice is HER2-targeted therapy. However, for HER2-negative patients there is no current 'obvious' chemotherapy choice. Ideally, clinicians would be able to identify patients with a high risk of relapse using chemotherapy, and they could then advise patients on the possibilities of taking part in clinical trials. As Fraser Symmans states, "the number of promising new treatments for breast cancer is growing rapidly, few patients currently participate in clinical trials of adjuvant treatments, trials are therefore slow to complete, and earlier access to such treatments for patient who have greatest potential to benefit may be delayed." A highly selective predictive tool would provide patients with a lot more certainty in their therapy decisions, and would also likely improve treatment compliance and outcome.

The elimination of detectable invasive or metastatic disease (pathologic complete response) is predictive of long-term survival in patients with breast cancer. Unfortunately, molecular tools to predict pathologic complete response have, until now, largely been disappointing. Factors such as age, tumor stage, and estrogen receptor status are still the standard, and molecular tools have not been tested using survival as an end point.

Against this background, investigators at the MD Anderson Cancer Center led by Symmans, initiated a prospective multicenter study to investigate genomic signatures to predict the success of sequential taxane and anthracycline-based neoadjuvant chemotherapy.

The researchers collected samples from patients prior to therapy and then

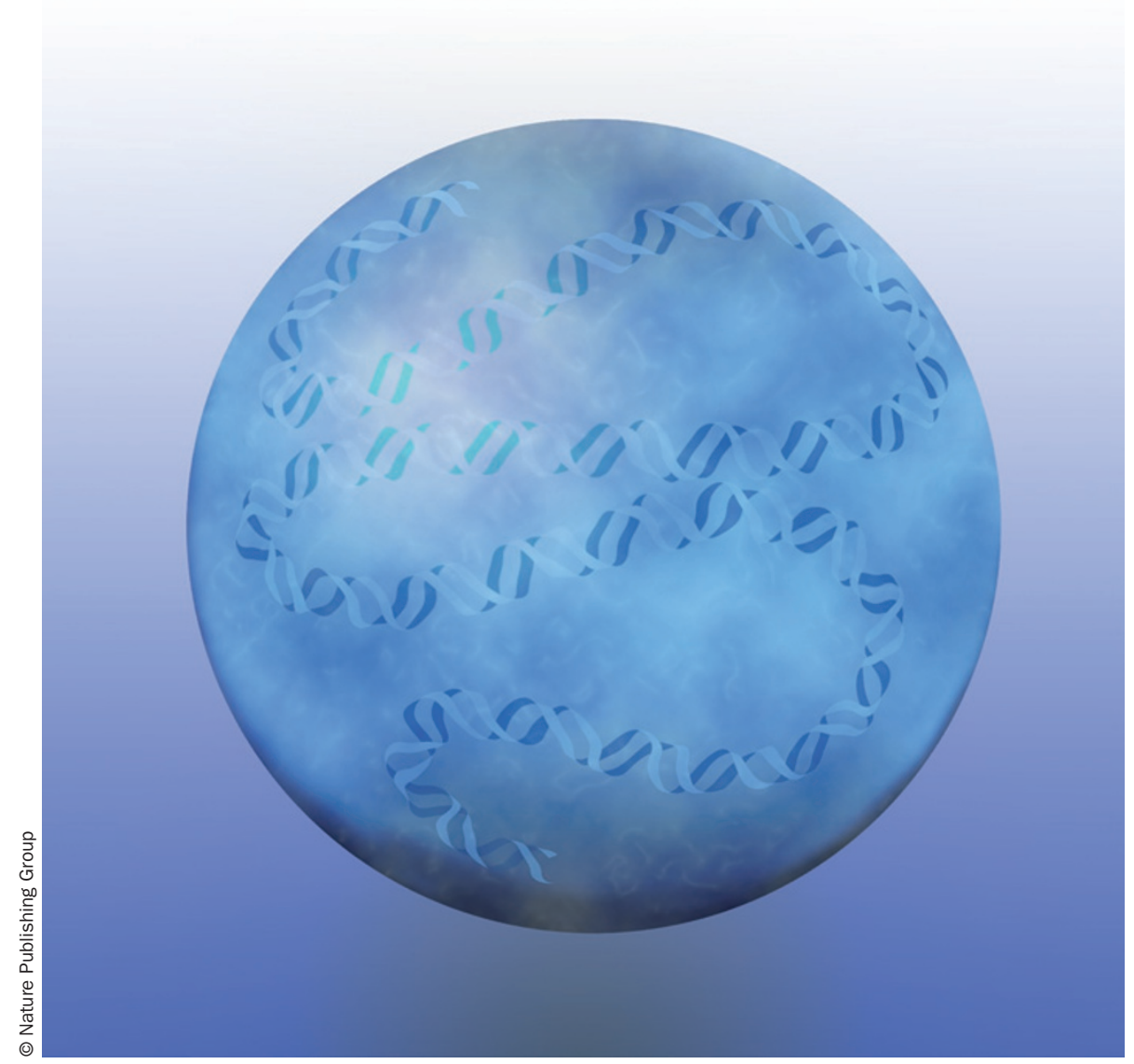

performed genetic analysis on those samples in conjunction with assessment of the long-term follow-up data. For the discovery cohort, 227 samples from patients with newly diagnosed HER2negative breast cancer were used to define genetic signatures for 'treatment sensitive' or 'treatment insensitive' patients.

"Separately training signatures to predict resistance and other signatures to predict excellent response enabled us to overcome potentially confounding and overlapping biology related to these extreme outcomes," Symmans comments.

Once the predictive test had been developed the researchers used the test in a validation group. "The test result of predicted treatment sensitivity was predictive of excellent pathologic response and high probability of survival without distant relapse or death... This is a significant advance beyond earlier published efforts to develop chemopredictive tests for which we found that there was actually a paradoxical relationship between predicted chemosensitivity and worse survival," explains Symmans.

Could it be possible to use this test to guide treatment, and take some of the guess work away? Further independent validation studies are already under way.

\section{Rebecca Kirk}

Original article Hatzis, C. et al. A genomic predictor of response and survival following taxane-anthracycline chemotherapy for invasive breast cancer. JAMA 18 , 1873-1881 (2011) 